\title{
MEASURE CONVERGENT SEQUENCES IN LEBESGUE SPACES AND FATOU'S LEMMA
}

\author{
HEINZ-ALBRECHT KLEI
}

\begin{abstract}
Let $\left(f_{n}\right)$ be a sequence of positive $P$-integrable functions such that $\left(\int f_{n} d P\right)_{n}$ converges. We prove that $\left(f_{n}\right)$ converges in measure to $\varliminf_{n \rightarrow \infty} f_{n}$ if and only if equality holds in the generalised Fatou's lemma. Let $f_{\infty}$ be an integrable function such that $\left(\left\|f_{n}-f_{\infty}\right\|_{1}\right)_{n}$ converges. We present in terms of the modulus of uniform integrability of $\left(f_{n}\right)$ necessary and sufficient conditions for $\left(f_{n}\right)$ to converge in measure to $f_{\infty}$.
\end{abstract}

\section{INTRODUCTION}

In [6] we proved the following result: let $(\Omega, \Sigma, P)$ be a probability space and $\left(f_{n}\right)$ a sequence of positive integrable functions such that $\left(\int f_{n} d P\right)_{n}$ converges. Then $\left(f_{n}\right)$ converges in norm to $\varliminf_{n \rightarrow \infty} f_{n}$ if and only if equality holds in Fatou's lemma. This is a striking example of the well known fact that under suitable extreme point conditions, weak convergence in $L^{1}$-spaces (and even much less) implies strong convergence [1]. By means of the modulus of uniform integrability of $\left(f_{n}\right)$ (to be defined later), we proved a generalisation of Fatou's lemma [6, Corollary 4]. In the present paper we pose the following question: when does $\left(f_{n}\right)$ converge in measure to $\underset{n \rightarrow \infty}{\lim } f_{n}$ ? We show that this is the case if and only if for all subsequences of $\left(f_{n}\right)$ equality holds in the generalised Fatou's lemma (Theorem 3). More generally we study the convergence in measure of a bounded sequence $\left(f_{n}\right)$ to an arbitrary element $f_{\infty} \in L^{1}(\mathbb{R})$ (Theorem 7 ). Both Theorem 3 and Theorem 5 enable us to give a straightforward proof of Lebesgue's convergence Theorem $[3, \mathrm{p} .122]$.

\section{Preliminaries}

Throughout this paper, $(\Omega, \Sigma, P)$ will be probability space. We shall consider the Banach space $L^{1}(\mathbb{R})$ of all (classes of) $P$-Bochner-integrable functions from $\Omega$ to $\mathbb{R}$.

Received 16 October 1995

Copyright Clearance Centre, Inc. Serial-fee code: 0004-9729/96 \$A2.00+0.00. 
In [7] Rosenthal defined the modulus of uniform integrability $\eta(H)$ of a bounded subset $H \subseteq L^{1}(\mathbb{R})$ : For $\varepsilon>0$, put

$$
\begin{aligned}
\eta(H, \varepsilon) & =\sup \left\{\int_{A}|h| d P: h \in H, A \in \Sigma, P(A) \leqslant \varepsilon\right\} \\
\eta(H) & =\lim _{\varepsilon \rightarrow 0^{+}} \eta(H, \varepsilon) .
\end{aligned}
$$

Thus $H$ is uniformly integrable if and only if $\eta(H)=0$.

\section{Results}

We start with a lemma proved in [4] and extended to Banach space valued integrable functions in [5].

LEMMA 1 . Let $f=\left(f_{n}\right)$ be a bounded sequence in $L^{1}\left(\mathbb{R}_{+}\right)$converging in measure to an element $f_{\infty}$ of $L^{1}\left(\mathbb{R}_{+}\right)$. Then the following assertions are equivalent:

(i) $\lim _{n \rightarrow+\infty} \int f_{n} d P=\eta(f)+\int f_{\infty} d P$ and $\eta\left(f^{\prime}\right)=\eta(f)$ for each subsequence $f^{\prime}$ of $f$

(ii) the sequence of reals $\left(\int f_{n} d P\right)_{n}$ converges in $\mathbb{R}_{+}$.

Corollary 2. Let $f=\left(f_{n}\right)$ be a bounded sequence in $L^{1}(\mathbb{R})$ converging in measure to $f_{\infty} \in L^{1}(\mathbb{R})$. Then $\left(\left\|f_{n}-f_{\infty}\right\|_{1}\right)_{n}$ converges in $\mathbb{R}$ if and only if $\eta\left(f^{\prime}\right)=$ $\eta(f)$ for each subsequence $f^{\prime}$ of $f$ and in this case $\lim _{n \rightarrow+\infty}\left\|f_{n}-f_{\infty}\right\|_{1}=\eta(f)$.

ThEOREM 3. Let $f=\left(f_{n}\right)$ be a bounded sequence in $L^{1}\left(\mathbb{R}_{+}\right)$such that the sequence $\left(\int f_{n} d P\right)$ converges in $\mathbb{R}_{+}$. Then the following assertions are equivalent:

(i) $\lim _{n \rightarrow+\infty} \int f_{n} d P=\eta(f)+\int \varliminf_{n \rightarrow \infty} f_{n} d P$ and $\eta\left(f^{\prime}\right)=\eta(f)$ for each subsequence $f^{\prime}$ of $f$;

(ii) the sequence $\left(f_{n}\right)$ converges in measure to $\varliminf_{n \rightarrow \infty} f_{n}$.

Proof: The implication (ii) $\Rightarrow$ (i) is a consequence of Lemma 1. Suppose now that (i) is true. Let $f^{\prime}=\left(f_{n}^{\prime}\right)$ be a subsequence of $f$. On account of the generalised Fatou's lemma [6, Corollary 4], we have

$$
\lim _{n \rightarrow+\infty} \int f_{n} d P \geqslant \eta(f)+\int \varliminf_{n \rightarrow \infty} f_{n}^{\prime} d P
$$

By comparing this inequality with the hypothesis, we obtain the following relation:

$$
\int \varliminf_{n \rightarrow \infty} f_{n} d P \geqslant \int \varliminf_{n \rightarrow \infty} f_{n}^{\prime} d P .
$$


It follows that $\varliminf_{n \rightarrow \infty} f_{n}=\varliminf_{n \rightarrow \infty} f_{n}^{\prime} P$-almost everywhere. Hence

$$
\lim _{n \rightarrow+\infty} \int f_{n}^{\prime} d P=\eta\left(f^{\prime}\right)+\int \varliminf_{n \rightarrow \infty} f_{n}^{\prime} d P .
$$

So Theorem 10 of [6] applies to the sequence $\left(f_{n}^{\prime}\right)$ and says that there is a further subsequence $\left(f_{n}^{\prime \prime}\right)$ of $\left(f_{n}^{\prime}\right)$ converging in measure to $\varliminf_{n \rightarrow \infty} f_{n}^{\prime}$, which equals $\varliminf_{n \rightarrow \infty} f_{n} P$ almost everywhere.

The proof is complete.

Proposition 4. Let $f=\left(f_{n}\right)$ be a bounded sequence in $L^{1}\left(\mathbb{R}_{+}\right)$and let $f^{\prime}=\left(f_{n}^{\prime}\right)$ a subsequence of $f$ such that $\underset{n \rightarrow+\infty}{\varliminf_{\rightarrow}} \int f_{n}^{\prime} d P=\varliminf_{n \rightarrow \infty} \int d P$. Then the following assertions are equivalent:

(i) $\varliminf_{n \rightarrow \infty} \int f_{n} d P=\eta\left(f^{\prime}\right)+\int \varliminf_{n \rightarrow \infty} f_{n} d P$ and $\eta\left(f^{\prime}\right)=\eta\left(f^{\prime \prime}\right)$ for each subsequence $f^{\prime \prime}$ of $f^{\prime}$;

(ii) the sequence $\left(f_{n}^{\prime}\right)$ converges in measure to $\varliminf_{n \rightarrow \infty} f_{n}$.

Proof: Suppose that (i) is true. Let $f^{\prime}=\left(f_{n}^{\prime}\right)$ be a subsequence of $\left(f_{n}\right)$ satisfying the hypothesis of Proposition 4. It follows that

$$
\lim _{n \rightarrow+\infty} \int f_{n}^{\prime} d P=\eta\left(f^{\prime}\right)+\int \varliminf_{n \rightarrow \infty} f_{n} d P \leqslant \eta\left(f^{\prime}\right)+\int \varliminf_{n \rightarrow \infty} f_{n}^{\prime} d P .
$$

By the generalised Fatou's lemma [6, Corollary 4] we obtain

$$
\lim _{n \rightarrow+\infty} \int f_{n}^{\prime} d P \geqslant \eta\left(f^{\prime}\right)+\int \varliminf_{n \rightarrow \infty} f_{n}^{\prime} d P .
$$

Thus we have two equalities in (1). Since all subsequences of $f^{\prime}$ have the same modulus of uniform integrability, Theorem 3 applies to the sequence $f^{\prime}$. Consequently $\left(f_{n}^{\prime}\right)$ converges in measure to $\varliminf_{n \rightarrow \infty} f_{n}^{\prime}$. Now $\varliminf_{n \rightarrow \infty} f_{n}^{\prime}$ and $\varliminf_{n \rightarrow \infty} f_{n}$ are comparable and their integrals coincident because of the second equality in (1). This means that $\varliminf_{n \rightarrow \infty} f_{n}^{\prime}(\omega)=$ $\varliminf_{n \rightarrow \infty} f_{n}(\omega) P$-almost everywhere.

Conversely, suppose that (ii) is true and let $f^{\prime}=\left(f_{n}^{\prime}\right)$ be a subsequence of $f$ such that

$$
\lim _{n \rightarrow+\infty} \int f_{n}^{\prime} d P=\varliminf_{n \rightarrow \infty} \int f_{n} d P .
$$

As $\left(f_{n}^{\prime}\right)$ converges in measure to $\varliminf_{n \rightarrow \infty} f_{n}$, we can apply the implication (ii) $\Rightarrow$ (i) of Lemma 1 to the sequence $\left(f_{n}^{\prime}\right)$, and the proof is done. 
Let us consider a special case of Theorem 3. If $\eta(f)=0$, then we obtain a result which was the starting point of our investigation. Note that it was used in the proof of Theorem 3.

THEOREM 5. Let $f=\left(f_{n}\right)$ be a bounded sequence in $L^{1}\left(\mathbb{R}_{+}\right)$. Then the following assertions are equivalent:

(i) $\left(\int f_{n} d P\right)$ converges in $\mathbb{R}_{+}$and $\lim _{n \rightarrow+\infty} \int f_{n} d P=\int \varliminf_{n \rightarrow \infty} f_{n} d P$;

(ii) $\left(f_{n}\right)$ converges in norm to $\varliminf_{n \rightarrow \infty} f_{n}$.

Proof: Suppose that (i) is true. By the generalised Fatou's lemma we have

$$
\lim _{n \rightarrow+\infty} \int f_{n} d P \geqslant \eta(f)+\int \varliminf_{n \rightarrow \infty} f_{n} d P \text {. }
$$

It follows that $\eta(f)=0$. We know from Theorem 3 that $\left(f_{n}\right)$ converges in measure to $\varliminf_{n \rightarrow \infty} f_{n}$. Note that a measure convergent and uniformly integrable sequence converges in norm.

Remark. As pointed out in [6], the combination of Theorem 5 and Fatou's lemma yields Lebesgue's convergence theorem [3, p.122].

Lemma 6. Let $f=\left(f_{n}\right)$ be a bounded sequence in $L^{1}(\mathbb{R})$ converging in measure to an element $f_{\infty}$ belonging to $L^{1}(\mathbb{R})$. Then the sequence $\left(\left\|f_{n}\right\|_{1}\right)$ converges if and only if $\left(\left\|f_{n}-f_{\infty}\right\|\right)$ does and in this case we have $\lim _{n \rightarrow+\infty}\left\|f_{n}-f_{\infty}\right\|_{1}=\eta(f)=$ $\lim _{n \rightarrow+\infty}\left(\left\|f_{n}\right\|_{1}-\left\|f_{\infty}\right\|_{1}\right)$.

Proof: We know from Brezis and Lieb [2] that

$$
\lim _{n \rightarrow+\infty}\left(\left\|f_{n}\right\|_{1}-\left\|f_{n}-f_{\infty}\right\|_{1}\right)_{n}=\left\|f_{\infty}\right\|_{1} .
$$

Suppose that $\lim _{n \rightarrow+\infty}\left\|f_{n}\right\|_{1}$ exists. As $\left(\left|f_{n}\right|\right)_{n}$ converges in measure to $\left|f_{\infty}\right|$, it follows from Lemma 1 of [4] that

$$
\lim _{n \rightarrow+\infty}\left\|f_{n}\right\|_{1}=\eta(f)+\left\|f_{\infty}\right\|_{1} .
$$

The combination of the last two equalities yields the first implication. To prove the opposite implication, suppose that the sequence $\left(\left\|f_{n}-f_{\infty}\right\|_{1}\right)_{n}$ converges. We know from Lemma 1 of [4] that its limit is $\eta(f)$. An application of Brezis' and Lieb's equality completes the proof.

THEOREM 7. Let $f=\left(f_{n}\right)$ be a bounded sequence in $L^{1}(\mathbb{R})$ and let $f_{\infty}$ be an element of $L^{1}(\mathbb{R})$. Suppose that $\left(\left\|f_{n}-f_{\infty}\right\|_{1}\right)_{n}$ converges in $\mathbb{R}$. Then the following assertions are equivalent:

(i) $\left(f_{n}\right)$ converges in measure to $f_{\infty}$; 
(ii) $\lim _{n \rightarrow+\infty}\left\|f_{n}-f_{\infty}\right\|_{1} \leqslant \eta(f)$ and $\eta(f)=\eta\left(f^{\prime}\right)$ for each subsequence $f^{\prime}$ of $f$.

(iii) $\lim _{n \rightarrow+\infty}\left\|f_{n}-f_{\infty}\right\|_{1}=\eta(f)$ and $\eta(f)=\eta\left(f^{\prime}\right)$ for each subsequence $f^{\prime}$ of $f$.

Proof: We know from Corollary 2 that (i) implies (iii). Suppose now that (ii) is true and let $f^{\prime}=\left(f_{n}^{\prime}\right)$ be any subsequence of $f$. Note that

$$
\lim _{n \rightarrow+\infty}\left\|f_{n}^{\prime}-f_{\infty}\right\|_{1} \leqslant \eta\left(f^{\prime}\right)
$$

Hence Theorem 6 of [4] applies to the subsequence $\left(f_{n}^{\prime}\right)$ and says that there is a further subsequence $\left(f_{n}^{\prime \prime}\right)$ of $\left(f_{n}^{\prime}\right)$ which converges in measure to $f_{\infty}$. Consequently assertion (i) follows.

PROPOSITION 8. Let $f=\left(f_{n}\right)$ be a bounded sequence in $L^{1}\left(\mathbb{R}_{+}\right)$such that

$$
\lim _{n \rightarrow \infty} \frac{1}{n} \sum_{k=0}^{n} \int f_{k} d P=\int \varliminf_{n \rightarrow \infty} f_{n} d P .
$$

Then the following statements hold:

(i) $\left(1 / n \sum_{k=0}^{n} f_{k}\right)_{n}$ converges in norm to $\varliminf_{n \rightarrow \infty} f_{n}$;

(ii) Let $f^{\prime}=\left(f_{n}^{\prime}\right)$ be any subsequence of $\left(f_{n}\right)$ satisfying $\lim _{n \rightarrow+\infty} \int f_{n}^{\prime} d P=$ $\varliminf_{n \rightarrow \infty} \int f_{n} d P$. Then $\left(f_{n}^{\prime}\right)$ converges in norm to $\varliminf_{n \rightarrow \infty} f_{n}$.

Proof: Put $m(f)=\left(1 / n \sum_{k=0}^{n} \int f_{k} d P\right)_{n}$. Note that

$$
\begin{aligned}
\int \varliminf_{n \rightarrow \infty} f_{n} d P=\lim _{n \rightarrow \infty} \frac{1}{n} \sum_{k=0}^{n} \int f_{k} d P & \geqslant \eta(m(f))+\int \varliminf_{n \rightarrow \infty} \frac{1}{n} \sum_{k=0}^{n} f_{k} d P \\
& \geqslant \eta(m(f))+\int \varliminf_{n \rightarrow \infty} f_{n} d P .
\end{aligned}
$$

The first of the preceding inequalities comes from the generalised Fatou's lemma. The second one is obvious. It follows that $\eta(m(f))=0$ and that $\varliminf_{n \rightarrow \infty} f_{n}(\omega)=$ $\varliminf_{n \rightarrow \infty} 1 / n \sum_{k=0}^{n} f_{k}(\omega) P$-almost everywhere. Now the hypothesis can be written as follows:

$$
\lim _{n \rightarrow \infty} \frac{1}{n} \sum_{k=0}^{n} \int f_{k} d P=\int \varliminf_{n \rightarrow \infty} \frac{1}{n} \sum_{k=0}^{n} f_{k} d P .
$$


Theorem 5 applies and yields the assertion (i).

Let $f^{\prime}=\left(f_{n}^{\prime}\right)$ be as in (ii). Note that

$$
\varliminf_{n \rightarrow \infty} f_{n} \int f_{n} d P \leqslant \lim _{n \rightarrow \infty} \frac{1}{n} \sum_{k=0}^{n} \int f_{k} d P=\int \varliminf_{n \rightarrow \infty} f_{n} d P .
$$

In particular we have $\underset{n \rightarrow \infty}{\lim } \int f_{n} d P=\int \varliminf_{n \rightarrow \infty} f_{n} d P$. On the other hand, we know that

$$
\varliminf_{n \rightarrow \infty} \int f_{n} d P=\lim _{n \rightarrow+\infty} \int f_{n}^{\prime} d P \geqslant \eta\left(f^{\prime}\right)+\int \varliminf_{n \rightarrow \infty} f_{n}^{\prime} d P \geqslant \eta\left(f^{\prime}\right)+\int \varliminf_{n \rightarrow \infty} f_{n} d P .
$$

So the preceding inequalities reduce to equalities and it follows that $\eta\left(f^{\prime}\right)=0$. Proposition 4 or Theorem 5 enable us to say that $\left(f_{n}^{\prime}\right)$ converges in norm to $\varliminf_{n \rightarrow \infty} f_{n}$.

\section{REFERENCES}

[1] E.J. Balder, 'On equivalence of strong and weak convergence in $L_{1}$-spaces under extreme point conditions', Israel J. Math. 75 (1991), 21-47.

[2] H. Brezis and E. Lieb, 'A relation between pointwise convergence of functions and convergence of functionals', Proc. Amer. Math. Soc. 88 (1983), 486-490.

[3] N. Dunford and J.T. Schwartz, Linear operators, Part I (Interscience Publishers, New York, 1962).

[4] H.-A. Klei, 'Convergences faible, en measure et au sens de Cesaro dans $L^{1}(\mathbb{R})$ ', C.R. Acad. Sci. Paris 315, Série I (1992), 9-12.

[5] H.-A. Klei, 'Convergence and extraction of bounded sequences in $L^{1}(\mathbb{R})$ ', J. Math. Anal. Appl. (to appear).

[6] H.-A. Klei and M. Miyara, 'Une extension du lemme de Fatou', Bull. Sci. Math. (2 ${ }^{e}$ série) 115 (1991), 211-222.

[7] H.P. Rosenthal, 'Sous-espaces de $L^{1}$ ', (Lectures held at the University Paris VI, 1979).

Université de Reims

Département de Mathématiques

Moulin de la Housse

B P 347

51062 Reims Cedex

France

e-mail: heinz.klei@univ-reims.fr 